\title{
Transiciones en el consumo de drogas en Colombia
}

\section{Transitions in drug abuse in Colombia}

Augusto Pérez Gómez*
*Corporación Nuevos Rumbos, Bogotá, Colombia. Director.

Enviar correspondencia a:

Augusto Pérez Gómez, Calle 108 A \# 4-15,Bogotá,

Colombia, Sur América.

E-mail: aperez@nuevosrumbos.org.

\section{RESUMEN}

Las transiciones en el consumo de sustancias (el paso de una sustancia a otra y de una vía de administración a otra) han sido un fenómeno poco estudiado en América Latina. En este trabajo se entrevistaron 96 personas (14 a 50 años de edad) en tratamiento en siete ciudades de Colombia; se identificaron cinco tipos de transición (general, acumulativa, sustitutiva, inversa y negativa) que ocurren en nueve momentos diferentes; se presentaron 113 'patrones transicionales' (combinaciones de dos sustancias), 23 de los cuales eran comunes a hombres y mujeres, 10 exclusivamente femeninos y 80 exclusivamente masculinos. Contra toda expectativa en ese grupo, seleccionado al azar, aparecieron 21 casos de consumo de heroína, principalmente en los niveles sociales alto y medio, más en hombres que en mujeres y en personas muy jóvenes: 11 de los 21 tenían menos de 20 años. Las principales transiciones involucran cuatro sustancias (marihuana cocaina, tranquilizantes e inhalables) y se presentan mayoritariamente en los menores de 23 años. Los jóvenes tienden a consumir marihuana, cocaína, tranquilizantes, pegantes, éxtasis, popper y heroína; los mayores cocaína, basuco y marihuana. Las mezclas, los cambios de sustancia y de vía de administración están siempre asociados a la búsqueda de efectos más potentes. Muchos jóvenes consideran que la marihuana es inofensiva o incluso benéfica, pues cura el cáncer que produce el cigarrillo; la heroína es considerada como la droga más peligrosa por sus efectos a todos los niveles.

Las principales limitaciones de este estudio son: el pequeño número global de sujetos, el pequeño número de mujeres y la restricción de entrevistas a personas que están actualmente en tratamiento.

\section{ABSTRACT}

Transitions in drug abuse (going from one substance to another and from one form of administration to another) has been scarcely studied in Latin America. In this project 96 people in treatment, aged 14 to 50, were interviewed in seven Colombian cities. Five kinds of transition were identified: general, cumulative, substitutive, reverse and negative, occurring at nine different points; 113 'transitional patterns' (combinations of two substances) were found, 23 common to men and women, 10 exclusive to women and 80 exclusive to men. Contrary to all expectations, 21 cases of heroin users appeared in this sample, mainly among upper- and middle-class users, more in men than in women, and in very young people: 11 out of 21 were under 20 years of age. The 10 main transitions only involve four substances: marihuana, cocaine, inhalants and tranquillizers, and are most common amongst those under age 23. The youngest use marihuana, cocaine, tranquillizers, glues, ecstasy, poppers and heroin; the eldest prefer cocaine, basuco and marihuana. Mixtures, changes of substance and changes of from of administration are always associated with a search for more powerful effects. Many youngsters believe that marihuana is either harmless or beneficial, because it can cure cancer caused by smoking tobacco. Heroin is considered the most dangerous drug because of its bad effects on all levels.

The main limitations of this study were: the small number of participants, the even smaller number of women, and the fact that only people in treatment were interviewed.

Key words: transitions, drug abuse, forms of administration, qualitative research. 


\section{INTRODUCCIÓN}

D esde el punto de vista sociológico el fenómeno de las transiciones es uno de los más sobresalientes en cuanto al consumo de drogas se refiere. Este concepto incluye básicamente dos fenómenos: el paso de un tipo de sustancia a otro: por ejemplo, de alcohol a marihuana, de marihuana a cocaína, de cocaína a heroína; y el paso de una forma de administración a otra: por ejemplo, de inhalar cocaina a inyectarse, o de fumar cocaina a inyectarse.

El tema comenzó a ser investigado formalmente en la década de los 80 , cuando algunos consumidores de marihuana y anfetaminas decidieron probar heroína y se encontraron involucrados en una serie de problemas de salud, que incluían desde infecciones provocadas por el hecho de compartir jeringas hasta el VIH/SIDA. El problema se agravó de manera especial en Italia, Escocia y algunas regiones de España, al igual que en la costa Este de los Estados Unidos. Poco tiempo después, la prohibición del consumo de opio fumado tradicional provocó un incremento sorprendente del consumo de heroína en paises como Tailandia, Myanmar y Vietnam; en este último país especialmente se produjo una oleada de infección por VIH que produjo la muerte de cientos de personas (Wilcox, Wagner y Anthony, 2002). Algo semejante ha venido ocurriendo recientemente en algunos paises de Europa Oriental y de Asia Central (Pakistán, Afganistán e Irán) (Wilcox, Wagner y Anthony ,2002). En los Estados Unidos, especialmente durante los años 90s, un número importante de cocaínómanos que usualmente aspiraban la cocaína por la nariz decidieron empezar a inyectársela; durante esa época se incrementó el número de emergencias hospitalarias asociadas con sobredosis.

En el caso colombiano, cierta información de carácter clínico (no suficientemente sistematizada y por ello de una confiabilidad limitada) da alguna idea sobre la transición de una sustancia a otra: lo que sabemos - o mejor, inferimos, pues nunca se han hecho estudios sistemáticos al respectoes que se pasa del alcohol a la marihuana, de esta a la cocaína y luego a otras sustancias (patrón conocido como "A-B-C": alcohol-'bareta'(marihuana)-cocaina); pero la realidad es que esa secuencia ha venido sufriendo cambios en los últimos años, debido a hechos como la aparición del éxtasis, el incremento del consumo de tranquilizantes o el aumento de la frecuencia del consumo de inhalables en clases económicamente pudientes. Igualmente, una cierta variedad de sustancias sintéticas como la quetamina, el 'cristal' y el 'popper' han surgido recientemente en el mercado colombiano y pueden haber influenciado ese supuesto patrón estándar.

En cuanto a la transición de vías de administración, sabemos que el consumo de basuco (cocaina fumada) ha disminuido (Rumbos, 2002; MPS/CICAD, 2005) y que un cierto número de personas ha comenzado a inyectarse heroína luego de haber estado fumándola por un cierto tiempo. Pero la realidad es que carecemos de información precisa y actualizada sobre esta temática.

Hace ya mucho tiempo Kandel (1975), propuso que las drogas legales son intermediarias necesarias entre el no uso y el consumo de marihuana. Los resultados de su estudio revelaban que mientras el $27 \%$ de los estudiantes de secundaria que fumaban y consumían alcohol iniciaban el consumo de marihuana entre $5-6$ meses después, solo el $2 \%$ de aquellos que no habían usado una sustancia legal, lo hacian. Así mismo, Kandel afirma que la marihuana es un paso crucial en el camino hacia las drogas ilícitas. Mientras que el $26 \%$ de consumidores de marihuana continuaban al consumo de LSD, anfetaminas o heroina, solamente 1\% de los no consumidores de marihuana y el $4 \%$ de los que usaban drogas legales (alcohol y cigarrillo) continuaron con tales sustancias. Estos resultados han sido corroborados por otros estudios (Kosterman, Hopkins, Guo, Catalano, y Abbot, 2000; Herrera, Wagner, Velasco, Borges y Lazcano, 2004).

Las etapas sugeridas por este autor revelaban ya desde ese entonces una clara secuencia en el uso de drogas: no uso, consumo de cerveza o vino, consumo de bebidas fuertes, consumo de cigarrillo; ninguno de los jóvenes que había consumido cerveza o vino progresaba a drogas ilícitas sin haber consumido bebidas fuertes o cigarrillo. Sin embargo, aunque los datos mostraran una secuencia en el uso de drogas, una droga particular no llevaría invariablemente al consumo de una de las drogas siguientes en la secuencia: "... muchos de los jóvenes pararon en una etapa particular de la secuencia y no progresaron más; otros regresaron a drogas más "bajas".

Lynskey, Heath, Bucholz, Slutske, Madde, Nelson et al. (2003) realizaron un estudio de consumo de marihuana con una muestra de 311 adultos gemelos (monocigóticos y dicigóticos), diferenciados por su uso temprano de marihuana (antes de los 17 años), y concluyeron que la iniciación temprana en el consumo de marihuana estaba asociada al aumento en los riesgos del uso de otras drogas y al abuso y la dependencia. Kandel y Davies (1992) evidenciaron la secuencia entre el alcohol y el cigarrillo, y el uso de marihuana y cocaína. Wilcox et al., (2002) se centran en la secuencia entre el uso de marihuana y el uso de LSD, PCP y otras drogas alucinógenas o "disociativas": su investigación muestra que los jóvenes usuarios de marihuana tienen mayores probabilidades de usar alucinógenos que los que no la consumen.

Por su parte, Wagner y Anthony (2002) han desarrollado estudios acerca de un posible mecanismo para comprender el proceso de verse involucrado en el uso de drogas: la exposición a oportunidades para usarlas. Ellos señalan que la mayor frecuencia en el uso de drogas entre hombres se relaciona con diferencias en la exposición a oportunidades para usar drogas entre sexos, y más recientemente ofrecen evidencia a favor de la idea de que los usuarios de alcohol o de tabaco tienen mayor riesgo de usar marihuana, cocaína y otras drogas precisamente porque en cada etapa del proceso tienen mayor exposición a oportunidades para usarlas. Igualmente, Crum, Lillie-Blanton y Anthony (1996) encontraron que la oportunidad de exposición al alcohol, cigarrillo y otras drogas depende en parte de las desventajas del contexto en el que se viva, mientras que Reboussin y Anthony (2001) encontraron relación entre los bajos niveles de supervisión de los padres y las posteriores transiciones de las primeras oportunidades al primer uso de alcohol, cigarrillo, marihuana e inhalables. 


\section{Transiciones en la vía de administración de una droga}

Existen diferentes vías de administración de una droga; por ejemplo, la heroina es frecuentemente fumada en "chino" (derivado del termino "chasing the dragon", tal y como se denomina en paises europeos al hecho de inhalar el humo de la heroína calentada sobre papel aluminio); asimismo la heroína se utiliza de modo intravenoso (más raramente de manera intramuscular o subcutánea), en ocasiones inhalada o fumada en forma de cigarrillo. La cocaina puede ser inhalada, fumada como pasta base 0 crack; también puede ser inyectada. Las anfetaminas son tomadas por vía oral, inhaladas e inyectadas. Strang, De Jarlais, Griffiths y Gossop (1992), consideran que las vías de administración de una droga varian en función de lugar y de tiempo: "...en ciudades como Londres, Manchester y Ámsterdam la heroína se consumía vía "chasing" o por inyección intravenosa. Mientras que en ciudades como Edinburgo y ciudades españolas e italianas, la vía intravenosa permanecía como la ruta de administración "universal" de la heroína. La vía inhalada aumenta de manera más evidente en ciudades como Nueva York...".

Auld, Dorn y South (1986) afirman que la disminución del costo de la heroína en los 80s en el Reino Unido fue uno de los factores que incidió en el aumento del uso de vías de administración que se denominaron "más costosas" (inhalación y fumar).

Según Strang, Heathcote y Watson (1987) las transiciones son progresiones en una pendiente deslizante, y se preguntan si es posible que los consumidores de drogas retrocedan en la pendiente; reportaron cómo una proporción de la cohorte había sido capaz de alcanzar una forma de recuperación parcial volviendo atrás en las vías de administración utilizadas. Según Stimson (1992) la existencia de una conciencia en salud del consumidor de droga, y la relevancia dada a las técnicas de inyección más seguras, son elementos que influyen en la naturaleza de continuar el uso de una droga y de la selección de su vía de administración. De otra parte, Oviedo, March, Romero y Sánchez (2005) afirman que en España el consumo de heroína por vía intravenosa ha ido disminuyendo, extendiéndose el uso de la misma por la vía pulmonar. El Observatorio Español sobre Drogas (2003) revela cómo en el año 1991 entre las personas admitidas a tratamiento por consumo de heroína, la vía intravenosa agrupaba al $74.7 \%$ de los usuarios, mientras que en el año 2001 se extendió solo al 24.2\%, prevaleciendo el consumo por inhalación o en forma de cigarrillos, con un 67.4\%. Sin embargo Oviedo et al., (2005) afirman que el uso de la vía pulmonar no descarta posibles transiciones hacia la inyectada, asociada ésta con una pérdida del control de consumo, marginación y mayor tiempo de consumo. Las mujeres que participaron en el estudio prefirieron la vía pulmonar, lo cual también ha sido indicado por otros autores (Gossop, Griffiths, y Strang, 1994). Los resultados sugieren que los más jóvenes y quienes se han iniciado más tardiamente en el consumo de heroína tienden a usar la vía inyectada en menor medida.

Para Oviedo et al., (2005) las explicaciones para el paso hacia el uso de la vía inyectada desde la pulmonar son: la presión social, tener una pareja que se inyecta, la influencia del mercado, cambios en las costumbres, entre otras. Según estos autores no se encontraron en su muestra evidencias de transiciones inversas, es decir, de usuarios por vía intravenosa a la vía pulmonar. En 1998 un 62,2\% de los tratados por dependencia de heroína eran fumadores y un 5,5\% 'esnifadores'. Los autores muestran que en los últimos años disminuyó de manera importante la práctica de inyectarse. De hecho, la proporción de tratados por heroína que usan esta droga preferentemente por vía parenteral descendió de 62,4\% en 1991 a 28,8\% en 1998.

La tendencia en otros paises desarrollados como el Reino Unido, Suecia y los EEUU, también puede ir en la misma dirección: por ejemplo, Swift, Maher y Sunjic (1999), encontraron que el $29 \%$ del grupo estudiado había pasado en el consumo de heroina, de fumar a inyectarse; los autores muestran que las transiciones "inversas" fueron poco comunes en la muestra estudiada. Ameijden y Countinho (2001) realizaron un estudio longitudinal en Ámsterdam y encontraron que la prevalencia de inyección en la muestra se redujo casi a la mitad (66\% al 36\%).

Tapia-Conyer, Cravioto, De la Rosa, Glavan y MedinaMora (2003) realizaron un estudio con 225 usuarios de drogas en la ciudad de Juárez (México), en el que encontraron que la principal vía de administración con que se inicia el uso de cocaína es la inhalada, y que la siguiente vía fue la inyectada, a diferencia del estudio de Khalsa, Anglin, Pareder, Potepan y Potter (1993) en el que la siguiente fue la vía fumada. Para Tapia-Conyer et al., (2003) estas diferencias pueden deberse a dos situaciones: la primera, a que existe una mayor disponibilidad de polvo de cocaina que de crack en esa zona de la frontera norte de México, y la segunda es "...que la cocaína y la heroína se están usando de manera simultánea y por vía intravenosa en el denominado 'speedball'...".

El análisis de la escasa literatura disponible sobre las transiciones muestra que se trata de un tema de enorme importancia para lograr una adecuada comprensión del fenómeno de las drogas, caracterizado por su inmensa variabilidad, por la permanente aparición de nuevas sustancias en el mercado, por la tendencia a que los jóvenes inicien su consumo cada vez más temprano y por las masivas implicaciones que tienen estas prácticas sobre las personas, sus familias y la sociedad como un todo.

La presente investigación se propuso abordar el tema de las transiciones de una sustancia psicoactiva a otra, y de una vía de administración a otra, en una población de cerca de 100 personas con una historia de consumo, la mayor parte de ellas actualmente en tratamiento, en 7 ciudades de Colombia.

Los objetivos de este estudio fueron los siguientes:

1. Identificar patrones generales y sustitutivos de transición del consumo de sustancias psicoactivas (SPA) en personas de 14 a 50 años, de diferentes niveles socio económicos en 7 ciudades colombianas.

2. Identificar los procesos sociales que acompañan esos cambios: influencia de amigos, curiosidad, problemas familiares. 
3. Examinar las transiciones de vías de administración para las principales sustancias (cocaína, heroína, alcohol y marihuana) y examinar las circunstancias y las razones del cambio.

4. Analizar las implicaciones de los tipos de transiciones en los ámbitos de salud, económico y social, entre otros.

\section{MÉTODO}

Se hicieron 96 entrevistas en profundidad en Bogotá (20); Cali (20); Medellín (20); Cúcuta (5); Pasto (11); Armenia (10); Neiva (10). La distribución de las personas entrevistadas por sexo y por grupo de edad puede observarse en la Tabla 1.

Tabla 1. Distribución de las entrevistas.

\begin{tabular}{cccc}
\hline EDAD & HOMBRES & MUJERES & TOTAL CASOS \\
\hline Gr 14-17 & 17 & 8 & 25 \\
Gr 18-23 & 18 & 4 & 22 \\
Gr 24-30 & 17 & 7 & 24 \\
Gr 31-40 & 13 & 1 & 14 \\
Gr $>40$ & 10 & 1 & 11 \\
\hline & 75 & 21 & 96 \\
\hline
\end{tabular}

El grupo total estuvo compuesto por 75 hombres y 21 mujeres que estaban en centros de tratamiento o acababan de salir de uno de ellos; el promedio de edad fue de 26 años, con rangos entre 14 y 50 . No hubo ninguna selección: los entrevistadores llegaban a los centros de tratamiento debidamente autorizados por sus directores, explicaban el proyecto y pedian a quienes desearan participar que firmaran un consentimiento informado.

\section{Procedimiento}

Se diseñó una entrevista semi-estructurada cuya finalidad era explorar la historia de vida de los sujetos participantes, en lo que a consumo de drogas se refiere (ver anexo). Se capacitó al equipo de entrevistadores y de transcriptores, y se elaboró el conjunto de categorías que se emplearon para el análisis de entrevistas.

Todas las entrevistas fueron grabadas en audio, transcritas y procesadas con el programa de computador OSR N6 para datos cualitativos. Para tal efecto, se diseñó una estructura con seis nodos de análisis, cada uno de los cuales contiene múltiples categorías. Una vez asignados los códigos a los diferentes párrafos de las transcripciones, se organizaron cuatro equipos de dos personas que analizaron con detalle y caso por caso cada una de las temáticas objeto de esta investigación. Cada equipo construyó matrices analíticas teniendo como ejes fundamentales el sexo, el grupo etáreo ${ }^{1}$ y la ciudad. El análisis de la mayor parte de los temas se llevó a cabo examinando caso por caso; en algunos casos se analizó el conjunto de manera global y se sacaron conclusiones igualmente globales.

Es importante precisar que dado el elevado número de entrevistas (inusual para un estudio de carácter cualitativo), en varias oportunidades fue posible hacer análisis cuantitativos que contribuyen a hacer más claros los resultados; sin embargo, el propósito no es sacar conclusiones generales porque la muestra no es representativa (en el sentido estadístico) de nada; pero en un sentido no estadístico, sí es altamente representativa de las personas que acuden a centros de tratamiento en busca de ayuda en Colombia.

\section{RESULTADOS}

Aun cuando en el estudio global se analizaron 5 temas (historia de consumo, interés por las drogas, transiciones, imaginarios y consecuencias del consumo), este artículo contemplará únicamente lo referente a las transiciones ${ }^{2}$.

La palabra "transiciones" se refiere al paso del uso de una sustancia a otra (por ejemplo, de marihuana a cocaina, de esta a basuco, de basuco a ansiolíticos). En este trabajo se identificaron cinco clases de transiciones: sustitutivas, acumulativas, negativas y s o inversas. Solo la última ha sido analizada en la literatura, por lo que se imponen unas definiciones muy puntuales:

1. Transiciones generales: son los patrones sucesivos de paso de una sustancia a otra.

2. Transición sustitutiva: es aquella en la que la persona suspende el uso de una sustancia y la reemplaza por otra.

3. Transición acumulativa: El paso de una sustancia a otra no implica el abandono del consumo de la primera.

4. Transición negativa: Se refiere a las transiciones que no ocurren o que ocurren con muy poca frecuencia.

5. Transiciones inversas: Son aquellas en las que una persona, después de haber abandonado una sustancia o una vía de administración, regresa a ella (por ejemplo, pasa de cocaína inhalada a cocaína fumada y luego vuelve a inhalarla).

Lo mismo ocurre con las vías de administración. Como se verá más adelante, en un solo patrón de consumo se pueden combinar todas estas formas de transiciones, que serán denominadas 'patrones transicionales'.

\footnotetext{
1 El criterio para organizar los grupos de edad se ajusta a los resultados de los estudios epidemiológicos. El primer grupo corresponde a los menores de edad y los dos siguientes a aquellos en los que tiende a concentrarse fuertemente el consumo; los dos últimos son más amplios en cuanto a los rangos de edad (10 años) porque en ellos el consumo disminuye.

2 El estudio completo fue publicado en forma de libro: Pérez Gómez, A. (2007). Transiciones en el consumo de drogas en Colombia. Bogotá: Dupligráficas (200 páginas). Puede consultarse en www.nuevosrumbos.org
} 
El alcohol no fue analizado separadamente porque en el $100 \%$ de los casos fue la sustancia que precedió cualquier otro uso de sustancias. Ningún sujeto dejó de consumir alcohol durante su historia de consumo.

En el grupo de 96 personas se presentó un total de 341 casos de transiciones de una sustancia a otra; los tres primeros grupos de edad realizaron el $79 \%$ (268) de todos los casos y los dos últimos grupos de edad el 21\% (73), observándose una diferencia significativa entre los grupos de edad 1,2 y 3 y los grupos 4 y $5(Z=3.077 ; p<0.001)$; $\sin$ embargo el grupo 2 es el grupo que más casos presentó con un $28 \%$ (95) del total de casos de transición.

Del total de los casos se pudieron extraer 113 patrones transicionales entre dos SPAs, entendiéndose como patrón transicional el paso de una sustancia a otra que se da en al menos un caso de la muestra (por ejemplo, marihuana a cocaína, ansiolíticos a cocaína, etc.). Estos patrones se presentaron en un máximo de 9 momentos diferentes a lo largo de la historia de consumo de cada individuo, es decir, en este grupo hubo personas que hicieron una sola transición (por ejemplo, de alcohol a inhalables), hasta algunos que hicieron 9 cambios; sin embargo, las transiciones se distribuyeron mayoritariamente entre 1 y 5 , siendo en la tercera transición donde se presenta mayor número de patrones transicionales (ver Figura 1).

De los 113 patrones transicionales obtenidos, se tomaron los 10 que se presentaron con mayor frecuencia dentro de la muestra y se observó que estos se dieron de manera exclusiva con interacciones entre marihuana, cocaina, ansiolíticos, basuco y pegante (ver Tabla 2). El patrón transicional "marihuana a cocaína" es el que más se presenta (63/96 ó $65.6 \%$ de la muestra), seguido por "cocaína a ansiolíticos" (16/96 ó $16.7 \%$ de la muestra) con una diferencia significativa entre estas dos $(Z=6.251$; $p<0.001)$. El grupo de edad en el que más se presenta el patrón transicional "marihuana a cocaína" es el grupo 2 (20/22 ó $90.9 \%$ del grupo), y este a su vez se presenta de manera mayoritaria (39/96 ó $40.6 \%$ de la muestra) con una diferencia significativa frente a su aparición en transiciones posteriores $(Z=7.048 ; p<0.001)$.

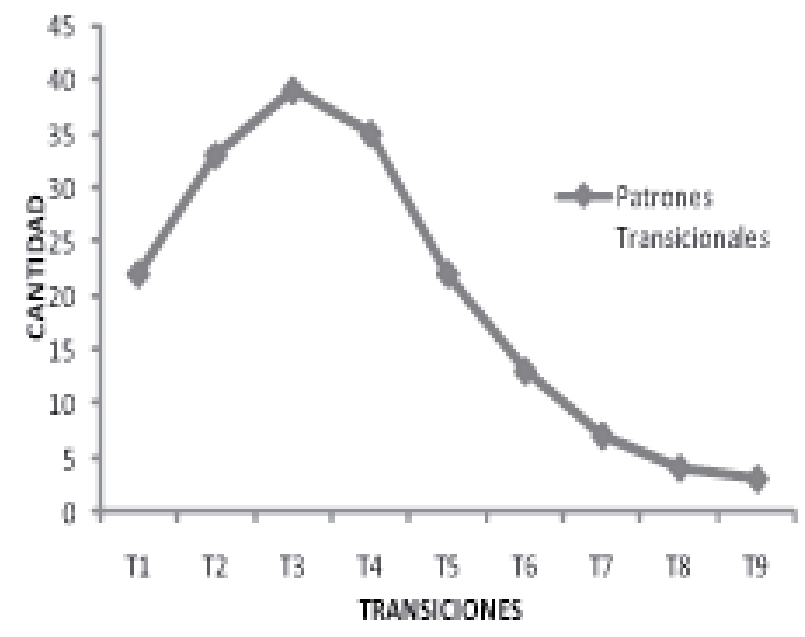

Nota: T1-T9 son las transiciones 1 a 9
Tabla 2. Patrones transicionales más frecuentes por grupos de edad.

\begin{tabular}{ccccccc}
\hline PATRÓN TRANSICIONAL & G1 & G2 & G3 & G4 & G5 & TOTAL \\
\hline Marihuana a cocaina & 16 & 20 & 13 & 9 & 5 & 63 \\
Cocaina a ansiolíticos & 4 & 2 & 6 & 4 & & 16 \\
Cocaina a marihuana & 3 & 3 & 9 & & & 15 \\
Marihuana a pegante & 9 & 4 & 2 & & & 15 \\
Marihuana a basuco & 1 & 1 & 5 & 3 & 3 & 13 \\
Cocaina a basuco & 1 & 2 & 2 & 5 & 1 & 11 \\
Pegante a marihuana & 3 & 3 & 3 & & & 9 \\
Marihuana a pepas & 1 & 3 & 2 & 2 & & 8 \\
Pegante a cocaina & 3 & 0 & 5 & & & 8 \\
Pepas a basuco & & 1 & 3 & 3 & 1 & 8 \\
\hline
\end{tabular}

Nota: $\mathrm{G} 1$ = 14-17 años; $\mathrm{G} 2$ = 18-23 años; $\mathrm{G} 3$ = 24-30 años; $\mathrm{G} 4$ = 31-40 años; $\mathrm{G} 5$ = > 40 años.

\section{Diferencias por sexo}

De las 341 casos observados, 280 fueron de hombres y 61 de mujeres, llegando a alcanzar un máximo de 9 transiciones en su historia de consumo para el caso de los hombres y un máximo de 7 para las mujeres; sin embargo, al discriminar el número de casos por grupos de edad se observó que para los hombres es el segundo grupo de edad donde se da el mayor número de casos (78/280 ó el 28\% del total de casos en hombres), mientras que para las mujeres, las del primer grupo de edad son las que tienen la mayor cantidad de casos (25/61 ó 40\% del total de casos en mujeres).

Se observó que de los 113 patrones que aparecieron de manera general para la muestra, 23 fueron mixtos (23/113 ó $20 \%$ del total de patrones de cambio), es decir, efectuados tanto por hombres como por mujeres, 80 fueron realizados exclusivamente por hombres (80/113 ó 71\%) y 10 exclusivamente por mujeres (10/113 ó 9\%).

El conjunto de patrones mixtos es donde se llevan a cabo la mayoría de casos de la muestra (210/341 ó $61.6 \%$ ). Aunque la diferencia en la cantidad de patrones exclusivamente masculinos y exclusivamente femeninos es significativa $(Z=10.25 ; p<0.001)$ de la misma manera en que lo es el número de casos que siguen estos patrones $(Z=11,396 ; p<0,001)$, cabe anotar que los 10 patrones exclusivamente femeninos aplicaron cada uno para un solo caso.

\section{Transiciones a heroína}

El consumo de heroína es un fenómeno bastante reciente en Colombia y con prevalencias de uso muy bajas (ligeramente superior al 1\%); sin embargo, en este estudio cuya selección de los centros de tratamiento estuvo basada únicamente en la benevolencia de sus directores para permitir entrevistar consultantes- aparecieron 21 personas (19 hombre y 2 mujeres, sobre un total de 96) que habian usado heroína o que eran adictos a ella. Tales cifras deben alertar a las autoridades sobre lo que puede ocurrir en un futuro muy cercano (ver Tabla 3).

Figura 1. Distribución de las entrevistas. 
Tabla 3. Consumo de Heroína por grupos de edad.

\begin{tabular}{ccc}
\hline & CASOS & $\%$ \\
\hline G1: 25 casos & 5 & 20,8 \\
G2: 22 casos & 8 & 36,6 \\
G3: 24 casos & 4 & 16,7 \\
G4: 14 casos & 2 & 14,3 \\
G5: 11 casos & 2 & 18,2 \\
\hline TOTAL: 96 casos & 21 & 22,1 \\
\hline
\end{tabular}

Nota: $\mathrm{G} 1=14-17$ años; $\mathrm{G} 2$ = 18-23 años; $\mathrm{G} 3$ = 24-30 años; $\mathrm{G} 4$ = 31-40 años; $\mathrm{G} 5$ = > 40 años.

\section{Transiciones sustitutivas y razones de cambio}

Se obtuvo información de 89 sujetos (68 hombres y 21 mujeres). De ellos, 27 aseguraron no haber hecho sustituciones, sino que acumulaban sustancias aun cuando no las usaran con la misma frecuencia o cantidad durante todo el tiempo.

Respecto a la distribución por sexos, es claro que los hombres hacen más sustituciones que las mujeres. En conjunto se dio un total de 38 sustituciones de SPA, de las cuales ninguna es exclusiva de las mujeres, mientras que los hombres presentan 27 sustituciones exclusivas.

Las sustituciones realizadas por las mujeres se caracterizan por:

1. Implicar, en la gran mayoría de los casos, el uso de marihuana, la cual en más de la mitad de las oportunidades es sustituida, principalmente, y en orden de importancia, por la cocaina y por el basuco.

2. Las ansiolíticos y el pegante son sustituidas únicamente por marihuana y cocaína, y esta sustitución se da exclusivamente por la influencia de otras personas y por las posibles consecuencias físicas producidas por la droga abandonada.

3. En las sustituciones que realizan las mujeres no se mencionan, ni como droga sustituida, ni como droga reemplazante, el éxtasis, los hongos o el crack, lo cual sí ocurre en el caso de los hombres.

4. En orden de importancia las principales razones para que las mujeres realicen una sustitución de droga son: el mayor placer que genera la nueva droga, las implicaciones sociales de la que abandona, la economía, el fácil acceso y la influencia de otras personas.

5. El promedio de edad en la que ocurren las sustituciones en la mujer es de 19 años y la edad en la que más mujeres afirman haber realizado una sustitución fue a los 18 años.

Las sustituciones en los hombres se caracterizan por:

1. Se encuentran mucho más dispersas en un mayor número de drogas, y la marihuana, a diferencia del caso de las mujeres, solo es mencionada por el $40 \%$ de los participantes y en más de la mitad de las oportunidades como sustancia reemplazada; sin embargo continúa siendo la sustancia más frecuentemente aludida.

2. No se presenta una sustitución de droga significativamente más frecuente entre los hombres: las que muestran el mayor número de casos, marihuana por cocaina y marihuana por pegante, agrupan cada una solo el 5.4\% del total de los casos.

3. Las principales razones que los hombres encuentran para llevar a cabo una sustitución de sustancia son, en primer lugar, la mayor sensación de placer que ofrecerian, y en segundo lugar, las consecuencias sociales que causarian en quienes las consumen.

4. El promedio de edad en el que ocurren las sustituciones en los hombres es de 20 años, y 16 años es la edad en la que más personas afirman haber hecho una sustitución.

5. La droga que se encuentra involucrada en mayor número de oportunidades en las sustituciones referidas por los entrevistados es la marihuana, seguida por la cocaína y en tercer lugar, las ansiolíticos (Rohypnol y otras benzodiacepinas).

\section{DISCUSION}

Las propuestas que siguen son, en estricto sentido, válidas únicamente para el grupo que participó en el estudio. Todas las generalizaciones son, por consiguiente, meras aproximaciones o fuentes de hipótesis. En este estudio se utilizaron como ejes de análisis el sexo, la edad, el nivel socioeconómico y la ciudad; las dos primeras resultaron relevantes para un gran número de elementos estudiados; las dos últimas, mucho menos.

En lo que se refiere a las transiciones, este trabajo permitió distinguir por lo menos cinco tipos de transiciones, de las cuales solo dos (la primera y la última) habian sido descritas en la literatura: generales, sustitutivas, acumulativas, negativas e inversas. En este estudio solo se analizaron las dos primeras; con respecto a la tercera, se constató que 27 personas, en mayor proporción mujeres, acumulaban sustancias; la gran mayoría eran jóvenes, pero no se analizaron razones porque los conceptos de 'acumulación' y 'sustitución' no existían en el inicio del estudio. Las transiciones negativas (las que no ocurren o son poco frecuentes: dos casos o menos en este estudio), fueron 140, sobre un total de 156 combinaciones posibles. Con respecto a las transiciones inversas no se obtuvo información sistemática, pero lo que se desprende de las transcripciones es que es un fenómeno poco frecuente.

De los 113 patrones transicionales, 23 fueron mixtos, 80 masculinos y 10 femeninos; el mayor número de casos se presentó entre los hombres de 18-23 años y entre las mujeres de 14 a 17; este último hecho podria explicarse diciendo que, aun cuando en general las mujeres se inician en las drogas más tarde que los hombres, una vez que lo hacen tienden a verse comprometidas rápidamente en 
problemas serios; esta hipótesis va en la dirección señalada por Stocco, Llopis, De Fazio, Facy, Mariani, Legl, Carvalho, Castillo y Rebollida (2000) y Stocco, Llopis, DeFazio, Calafat y Mendes (2002).

El hecho de que 64\% de los casos de consumo de SPA ilegales esté precedido por marihuana confirma los hallazgos de Kosterman et al. (2000) y de Herrera et al (2004).

De manera general, se encontró que el mayor número de casos de transición tuvo lugar en los 3 primeros grupos de edad, y especialmente en el grupo de 18-23 años, lo que permite inferir (y el análisis de los datos lo confirma) que es en estos grupos en los que se presenta una mayor tendencia a probar diferentes sustancias. Probablemente esto sea consistente con ciertas tendencias de la sociedad contemporánea, que incluyen el promover el máximo de experiencias, a ensayar continuamente cosas nuevas y el exceso de tolerancia y permisividad.

Es posible que la razón por la que las mujeres tienden a hacer un número menor de transiciones que los hombres esté asociado a un cierto temor a las consecuencias y al deseo de no tomar demasiados riesgos. Este tema requiere una exploración especifica.

Los 10 patrones transicionales más frecuentes fueron patrones que incluian interacciones entre pegante, marihuana, cocaína y ansiolíticos, y que se presentaban en su gran mayoría en las 3 primeras transiciones de la historia de consumo. Es posible que las transiciones y, por consiguiente, la historia de consumo de los individuos, guarde un fuerte vínculo con la oferta del mercado en la medida en que al parecer, las personas consumen lo que haya o lo que les sea más fácil de conseguir en su entorno.

Los patrones transicionales exclusivamente femeninos son pocos y cada uno de ellos se da sólo con un caso dentro de la muestra, lo que indicaría que las mujeres guian su consumo a partir de los patrones transicionales mixtos. Esta hipótesis deberá ser sometida a verificación en muestras más grandes de personas consumidoras, pero si se confirma podría ayudar a predecir el comportamiento de las mujeres en este tema.

Un aspecto que llama la atención, y que deberá profundizarse en estudios posteriores, es que la frecuencia de aparición de transiciones a ansiolíticos (cocaína a ansiolíticos y marihuana a ansiolíticos) es mayor en mujeres que en hombres. Es importante tener en cuenta que en general las mujeres muestran una mayor tendencia a tomar medicamentos; por otra parte, muchos estudios muestran (DNE, 1996; Rumbos, 2002; MPS/CICAD, 2005) que las mujeres consumen más tranquilizantes que los hombres y que si bien este dato en el pasado se refería a mujeres mayores de 40 años, en la actualidad se observa la misma tendencia de consumo en mujeres adolescentes.

La mayoría de los casos de transición a heroína se presentaron en los dos primeros grupos de edad (13 sobre 21. De todas formas, no debe perderse de vista que la edad de inicio promedio para el consumo de heroína es de más de 6 años por encima del promedio de la edad de inicio para marihuana. Es muy posible que si el problema se incrementa, en el futuro próximo la principal razón para pasar de heroína fumada a inyectada en quienes se vuelvan dependientes de esta sustancia, pase a ser económica: con la misma cantidad de heroina que sirve para una dosis fumada, se pueden hacer entre 5 y 10 de heroína inyectada.

Tal y como se afirmó en el marco teórico (Barrio y cols, 1997), 18 de los 21 consumidores de heroína de este estudio tenian una larga historia previa de consumo de cocaína; todos ellos tenian, en realidad, una historia prolongada (incluso los más jóvenes, aun cuando obviamente en tiempos más cortos) de consumo de otras sustancias.

Contrariamente a lo que se observa en Europa (Ameijden y Countinho, 2001; March, Romero y Sánchez, 2005) en Colombia la tendencia es a fumar heroína primero y a inyectarse después; esto se encuentra probablemente relacionado con los bajos costos y la disponibilidad.

La heroína es la sustancia que más sustituye a otras por el placer que produce y por el elevado grado de dependencia que genera (las personas dicen que no tienen recursos ni interés por otras sustancias). Seis transiciones a heroína fueron desde ansiolíticos: esto requiere mayor profundización.

Las dos razones básicas para hacer mezclas son la curiosidad y la búsqueda de contrarrestar efectos de otra sustancia. Quienes no hacen mezclas dicen que tienen temor a una mala reacción, no han pensado en esa posibilidad, o no quieren dañar los efectos de lo que consumen; esta última razón la dan quienes se han 'especializado' en una sustancia.

Conviene estudiar las transiciones negativas, acumulativas e inversas para tener una visión integral que dé lugar a nuevas predicciones y facilite el diseño de estrategias de prevención. Las transiciones negativas aparecen como poco frecuentes: así lo habian observado Oviedo et al (2005) y Swift, Mayer y Sunjic (1999).

La gran mayoría de los resultados de este estudio no tienen antecedentes en la literatura, por lo que no es posible hacer las comparaciones usuales. Como puede verse, la estrategia empleada permite generar una enorme cantidad de hipótesis, y en ese sentido este trabajo constituye una fuente seminal para investigaciones más detalladas; y esa también es su mayor limitación: las conclusiones no pueden generalizarse más allá de la población que fue entrevistada.

\section{CONCLUSIONES}

Los objetivos de este estudio se cumplieron a cabalidad. Aun cuando la literatura sobre el tema es escasa, los resultados coinciden con las conclusiones de los principales estudios en el área. La lectura de los resultados y de la discusión abre las puertas para estudios mucho más detallados y que se fundamenten en metodologías cuantitativas. 


\section{AGRADECIMIENTOS}

Este estudio fue financiado en su totalidad por la Dirección Nacional de Estupefacientes de Colombia.

Adicionalmente, esta investigación se llevó a cabo con la colaboración y participación de Marcela Correa, Jorge Rodríguez, Diana Raquel Sierra, Liliana González, Viviana Quintero, Paula Castillo, Angela Trujillo y Maria Constanza Lozano

\section{REFERENCIAS}

Ameijden, E. y Countinho, R. (2001). Large decline in injecting drug use in Amsterdam, 1986-1998: explanatory mechanisms and determinants of injecting transitions. Journal of Epidemical Community Health, 55, 356-363.

Auld, J., Dorn, N. y South, N. (1986). Irregular work, Irregular pleasures: heroin in the 1980s. in R. Matthews y J. Young, editores. Confronting Crime. London: Sage.

Crum, R.M., Lillie-Blanton, M. y Anthony, M.J. (1996). Neighborhood environment and opportunity to use cocaine and other drugs in late childhood and early adolescence. Drug and Alcohol Dependency, 43, 155-161.

Gossop, M., Griffiths, P. y Strang, J. (1994). Sex differences in patterns of drug taking behavior. A study at a London community drug team. British Journal of Psychiatry, 164, 101- 4.

Herrera, F., Wagner, M., Velasco, A., Borges, T. y Lazcano, S. (2004). Inicio en el consumo de alcohol y tabaco y transición a otras drogas en estudiantes de Morelos, México. Salud Pública de México, 132 - 140.

Kandel, D. (1975). Stages in adolescent involvement in drug use. Science, 190, $912-914$.

Kandel, D. y Davies, M. (1992). Progression to regular marijuana involvement: phenomenology and risk factors for near daily use. En M. Glantz y R. Pickens (eds.). Vulnerability to Drug Abuse. Washington, D.C. American Psychological Association

Khalsa, M., Anglin, D., Pareder, A., Potepan, P. y Potter, C. (1993). Pretreatment natural history of addiction: Preliminary 1-year follow up results. En: Tims F, Leukefeld $C_{1}$ (eds.). Cocaine Treatment: Research and Clinical Perspectives (pp. 218-235). National Institute on Drug Abuse Research Monograph.

Kosterman, R., Hopkins, J., Guo, J., Catalano, R y Abbot, R. (2000). The dynamics of alcohol and marijuana initiation: Patterns and predictor of first use in adolescence. American Journal of Public Health, 90, 360-366.

Lynskey, M., Heath, A., Bucholz, K., Slutske, W., Madde, P., Nelson, E., Statham, D. y Martin, N. (2003). Escalation of Drug use in EarlyOnset Cannabis Users vs. Co-twin Controls. JAMA, 289, 22-29.

MPS/CICAD (2005). Encuesta Nacional sobre consumo de sustancias psicoactivas en jóvenes escolarizados de 12 a 17 años en Colombia. Bogotá: MPS.

Naciones Unidas (2003) Informe Mundial sobre Drogas, Volumen 1: Análisis. Oficina de las Naciones Unidas contra la Droga y el Delito (ONUDD)

Naciones Unidas (2004). Informe Mundial sobre Drogas, Volumen 1: Análisis. Oficina de las Naciones Unidas contra la Droga y el Delito (ONUDD)
Naciones Unidas (2005). Informe Mundial sobre Drogas, Volumen 1: Análisis. Oficina de las Naciones Unidas contra la Droga y el Delito (ONUDD)

Naciones Unidas (2006). Informe Mundial sobre Drogas, Volumen 1: Análisis. Oficina de las Naciones Unidas contra la Droga y el Delito (ONUDD)

Naciones Unidas (2007). Informe Mundial sobre Drogas, Volumen 1: Análisis. Oficina de las Naciones Unidas contra la Droga y el Delito (ONUDD)

Naciones Unidas (2006). Jóvenes y drogas en paises sudamericanos: un desafio para las políticas públicas Primer estudio comparativo sobre uso de drogas en población escolar secundaria de Argentina, Bolivia, Brasil, Colombia, Chile, Ecuador, Paraguay, Perú y Uruguay.

Plan Nacional Sobre Drogas (2003). Informe No 6. Observatorio Español sobre Drogas. Ministerio de Sanidad y Consumo, Madrid.

Oviedo, E., March, J., Romero, M. y Sánchez, E. (2005). Factores asociados al uso de la vía pulmonar e intravenosa en una muestra de consumidores de heroina en Granada. Revista Española de Salud Pública, 79, 391-401.

Stenbacka, M., Allebeck, P. y Romelsjo, A. (1993). Initiation into drug abuse: the pathway from being offered drugs to trying cannabis and progression to intravenous drug abuse. Scandinavian Journal Social Medicine, 21, 31-39.

Stimson, G. (1992). Minimizing harm from drug use. En Heathcote, S. y Watson, P. (1987). Habit moderation in injecting drug addicts. Health Trends, 19, 16-18.

Stocco, P., Llopis, J., De Fazio, L., Facy, F., Mariani, E., Legl, T., Carvalho, M., Castillo, A. y Rebollida, M. (2000). Women and opiate addiction: a European perspective. IREFREA. Palma de Mallorca: España

Stocco, P., Llopis, J., DeFazio, L., Calafat, A. y Mendes, F. (2002). Women drug abuse in Europe: gender identity. IREFREA. Palma de Mallorca: España.

Strang, J., Des Jarlais, D., Griffiths, P. y Gossop, M. (1992). The study of transitions in the route of drug use: the route from one route to another. British Journal of Addiction, 87, 473-483.

Swift, W., Maher, L. y Sunjic, S. (1994). Transition between routes of heroin administration: a study of Caucasian and Indochinese heroin users in southwestern Sydney, Australia. Addiction, 94, 71-82.

Tapia-Conyer, R., Cravioto, P. De la Rosa, B, Glavan, F. y Medina-Mora, M. (2003). Historia natural del consumo de la cocaína: el caso de ciudad Juárez, Chihuahua. Salud Mental, 26, 12 - 21.

Wagner, F. y Anthony J. (2002). Into the world of illegal drug use: Exposure opportunity and other mechanisms linking alcohol, tobacco, marijuana, and cocaine use. American Journal of Epidemiology, 155, 918 - 925.

Wilcox, H., Wagner, F. y Anthony, J. (2002). Exposure opportunity as a mechanism linking youth marijuana use to hallucinogen use. Drug and Alcohol Dependence, 66, 127-135. 